\title{
Solving Transshipment Problem using EAs: How Good are the Solutions?
}

\author{
Ruhul Sarker* Non-member \\ Akira Namatame** Member
}

\begin{abstract}
Evolutionary Algorithms have attracted increasing attention in recent years, as powerful computational techniques, for solving many complex real-world problems. The successful application of Evolutionary algorithms to optimization problems is dependent on the methods and parameters used for the algorithms. In this paper, we develop three evolutionary algorithms for solving a transshipment problem. We investigate the effect of population sizes on the quality of solutions to be obtained, the computational time to be required and the size of search spaces of the problems under consideration. We also use a well-known conventional optimization package to compare the quality of solutions. The numerical results are analyzed and the interesting findings are discussed.
\end{abstract}

Keywords: evolutionary algorithms, optimization, transshipment problem.

\section{Introduction}

Evolutionary Algorithms (EAs) have shown tremendous success in solving complex real-world problems ${ }^{(6)(3)}$ over the last two decades. Due to its parallelism and some intelligent properties such as self-organization, adaptation and self-learning, EAs have been applied successfully to many problems where the classical approaches are either un-available or generally lead to unsatisfactory results. In recent years, the interests in EAs have been growing dramatically ${ }^{(28)(29)}$.

EAs are basically heuristic search algorithms. The two major steps in applying any heuristic search algorithm to a particular problem are the specification of the representation and the evaluation (i.e, fitness) function. These two items work like a bridge between the original problem context and the problem-solving framework. When defining an evolutionary algorithm, one needs to choose its core components, such as variation operators (mutation and recombination) that suit the representation, selection mechanisms for selecting parents and survivors, and an initial population. Each of these components may have their own parameters, for instance: the probability of mutation, the tournament size of selection or the population size. The values of these parameters greatly contribute to: whether the algorithm will find an acceptable solution, and whether it will find such a solution efficiently. Choosing the right parameter values, however, is a time-consuming task and considerable effort has gone into developing good heuristics for it ${ }^{(5)}$.

In this paper, we have developed a simple genetic algorithm for solving a transshipment problem. To improve

\footnotetext{
* School of Information Technology and Electrical Engineering, University of New South Wales at ADFA, Canberra, Australia ** Department of Computer Science, National Defence Academy, Japan
}

the quality of solutions, we are interested to find an appropriate population size of the problem under consideration. However, the question as to how to choose an adequate population size for a particular domain is difficult and has puzzled practitioners for a long time ${ }^{(25)(1)}$ (14) (11) (12). If the population size is too small, it is not likely that the GAs will find solutions of high quality. However, if the population size is too large, the GAs will unnecessarily waste processing time leading to unacceptably slow convergence. Harik et al ${ }^{(14)}$ exploited the similarity between the gambler's ruin (one-dimensional random walk) problem and the selection mechanism of GAs for determining an adequate population size that guarantees a solution of the desired (/target) quality. In their work, they assumed that mutation is not a dominant operator. The use of many domain-dependent variables and variable-length cromosomes makes their populationsizing equation unsuitable for applying to other problem domains ${ }^{(1)}$. However, the general idea is that the population size may be increased to reach a particular quality of solution.

Sakawa and Kato ${ }^{(20)}$ proposed a GA for solving a multidimensional 0-1 knapsack problem and compared the performance with a well-known conventional optimization technique such as branch-and-bound (B\&B). As they experimented with fixed population size, GAs provided optimal solutions for smaller problems and the quality of solution deteriorated (slowly) as the size of the problem instance increases. However, the computational time, for GA compared to $\mathrm{B} \& \mathrm{~B}$, is more and more appreciable as long as the size of the problem instance increases. Sarker et al (21) developed a GA for solving a constrained integer-nonlinear programming model arose from a joint product-raw material batch sizing problem. In their experimental study, they considered both real and binary coded GA with a number of different crossover, mutation and penalty function methods. 
They reported that, for any combination of the parameters and methods, the number of optimal solutions per run had been increased, for such a fixed size model, with the increase of the population sizes. From that study, it was also found that the number of optimal solutions per run had been decreased with the tightness of the constraints ${ }^{(21)(22)}$.

Vignaux and Michalewicz (27) developed a GA based methodology for solving a transportation problem. They used the transportation problem as a test case to investigate the relationship between representation structures and genetic operators for constrained optimization. Karanta et al (18) applied genetic algorithms successfully for solving a NP hard wood transportation problem. Thangiah and Nygard (26) improved the solution of multi-commodity trasshipment problem (MCTP) by integarting genetic algorithm with a MCTP solver. Jiang et al ${ }^{(17)}$ proposed a location model, which is a transportation model based GA, for physical distribution centers. Their model provided better solutions than the Alternate Location-Allocation (ALA) method. Gottlieb and Paulmann ${ }^{(13)}$ presented two genetic algorithms for solving fixed charge transportation problem. It is known that the main problem in designing GAs for constrained problems is the right choice of a proper constraint handling techniques. They investigated the suitability of two prominent techniques - the decoder approach and direct solution encoding with specialized operators to maintain feasibility. As they reported, the later one demonstrated its clear superiority.

Syarif and Gen ${ }^{(23)}$ and Syarif et al ${ }^{(24)}$ recently developed a spanning tree-based GA, with Prufer number representation, for solving different network optimization problems. Syarif and Gen ${ }^{(23)}$ applied the algorithm for solving a two-stage transportation problem. Although the problem is similar to our case problem, they differ in terms of mathematical formulation and GA applied to solving the model. Syarif et al ${ }^{(23)} \mathrm{im}-$ plemented the developed algorithm in solving a complex multi-stage logistics problem. Their limited experiments with the population sizes confirm the general idea of quality improvement. Gen et al ${ }^{(8)(9)}$ also extended the above single objective algorithm for solving bi-criteria transportation problem.

None of the above research papers explored the effect of population sizes on the search space, quality of solution and the computational time in general term. In this paper, in addition to the development of a simple GA, we will present experimental study to show the relationship between population sizes and quality of solutions under different search spaces. The number of fitness evaluations and the computational time required will also be analyzed. We will also solve the model using a well-known optimization package to compare the quality of solutions. To the best of our knowledge, no such study for a general optimization problem (like a transshipment problem) has been appeared in the literature. The experimental results confirm, the general idea, that the population sizes may need to increase (up to certain level) for improving the quality of solutions for a transshipment problem, and it may need further increasing for higher dimensional models. The relationship between the population size and the number of fitness evaluation is not linear.

The organization of the paper is as follows: following the introduction, this paper presents a simple transshipment problem and its mathematical model in section 2 . Section 3 develops genetic algorithms for solving the model developed in section 2. Section 4 reports and analyzes the computational results. Finally, the conclusions are provided.

\section{A Simple Transshipment Problem}

Consider a transshipment problem where $n$ sources, $m$ transshipment points and $r$ destinations exist. The sources have limited supply, the transshipment points have limited capacity to handle the supplied goods and the destinations have known demand. All the supplies from the sources are transported to the destinations via the transshipment points. The problem is to minimize the overall transportation cost while satisfying supply, capacity, demand and flow constraints. This problem can easily be formulated as a mathematical programming model as shown below.

We use the following notations in formulating the mathematical model:

$x 1_{i j}=$ The quantity transported from source $i$ to transshipment point $j$

$x 2_{j k}=$ The quantity transported from transshipment point $j$ to destination $k$

$C 1_{i j}=$ Unit transportation cost from source $i$ to transshipment point $j$

$C 2_{j k}=$ Unit transportation cost from transshipment point $j$ to destination $k$

$S_{i} \quad=$ Total supply from source $i$

$C P_{j} \quad=$ Capacity of transshipment point $j$

$D_{k} \quad=$ Demand of destination $k$

The mathematical model of the problem can be formulated as follows:

Minimize

$$
Z=\sum_{i} \sum_{j} C 1_{i j} x 1_{i j}+\sum_{i} \sum_{j} C 2_{j k} x 2_{j k} \cdots
$$

Subject to

$$
\begin{aligned}
& \sum_{j} x 1_{i j} \leq S_{i}, \quad \forall i \ldots \ldots \ldots \ldots \ldots \ldots \ldots \ldots \ldots \\
& \sum_{i} x 1_{i j} \leq C P_{j}, \\
& \sum_{j} x 2_{j k} \geq D_{k}, \quad \forall k \\
& \sum_{i} x 1_{i j}-\sum_{k} x 2_{j k} \geq 0, \quad \forall j \\
& x 1_{i j}, x 2_{j k} \geq 0 \text { and integer, } \forall i, j, k
\end{aligned}
$$

The objective of the problem is to minimize the overall transsipment costs. The first, second and third constraints ensure the supply limitation, transshipment 
point capacity and demand requirements respectively. The fourth or the flow constraint indicates that the total supply (in the second stage) from an transshipment node $j$ to all the destinations must be less than or equal to the supply (in the first stage) received by that $j$ from all sources. This is a standard integer programming model as the variables $x 1_{i j}$ and $x 2_{j k}$ are integer in nature. We assume there exist a number of feasible solutions. For any feasible solution, the first and the last two constraints are binding which makes it a difficult model to be solved by using EAs.

In this paper, we have chosen GAs to solve the above model as they are more popular among the practitioners. The transshipment problem was selected mainly for three reasons: (i) the problem arises in many logistics and transportation situations, (ii) this problem generates many tighter constraints which are difficult to handle by many algorithms and (iii) the corresponding mathematical model can be solved using a standard optimization package to compare the solutions.

\section{Development of GA}

We consider a simple real-coded GA, where the variable values were generated as real or integer numbers not as binary-string. Usually the binary coding is recognized as the most suitable encoding for any problem solution because it maximizes the number of schemata being searched implicitly (15) (10) but there have been many examples in the evolutionary computation literature where alternative representations have resulted in algorithms with greater efficiency and optimization effectiveness when applied to identical problems (see e.g. articles by Back and Schwefel (2) and Fogel and Stayton ${ }^{(7)}$, among others). Davis ${ }^{(4)}$ and Michalewicz (19) comment that for many applications real-values or other representations may be chosen to advantage over binary coding. There does not appear to be any general benefit in maximizing implicit parallelism in evolutionary algorithms, and therefore forcing problems to fit into a binary representation may not be recommended. As experimented by Sarker et al ${ }^{(21)(22)}$, real coded GAs perform better than Binary coded GAs for integer programming problems.

The first step of the algorithms is to generate an initial population. In this process, each variable (such as $x 1_{i j}$ and $x 2_{j k}$ as refer to our model above) of a candidate solution (i.e., an individual of the initial population) is generated randomly satisfying certain conditions as discussed later. Then the fitness for each individual is calculated and they are ranked from best to worst fitness values. The fitness function, used in our algorithm, may include penalty function to handle the constraints for constrained optimization. A tournament selection type strategy is used for selection as parents. All three algorithms used similar crossover to produce offspring. Mutation is not applied to the 2nd and 3rd algorithms as they require very specialized treatment to maintain feasibility. The details of initial population, selection, constraint handling, crossover and mutation are briefly described in the following subsections. The algorithms are terminated when the differences of best fitness values are zero over five consecutive generations.

The following characteristics were considered arbitrarily (as of Sarker et al ${ }^{(21)(22)}$ ) in running the Simple GA (SGA).

\subsection{Algorithm 1: Simple GA}

- Population size 50

- Initial population was generated randomly within the given range of supply, capacity and requirements.

- Heuristic crossover and non-uniform mutations

- Probability of crossover $=1.0$, and Probability of mutation $=$ up to 0.10

- Penalty methods for constraints handling: dynamic

- Selection for crossover: First rank all the individuals based on the objective function values. Then select randomly one from the top 10 and two from the rest. Crossover was made between the first one and the better of other two.

In conventional optimization, the penalty method converts the constrained problem into an equivalent unconstrained problem and then solves it using a suitable search algorithm. In general a penalty function approach places the constraints into the objective function via a penalty parameter in such a way that it penalizes any violation of the constraints. The modified problem is known as a penalty problem. In genetic algorithms, the penalty function method is used to transform the constrained problem into an unconstrained problem, the way we do in conventional optimization techniques. After converting the problem, GAs apply population based stochastic search procedures that are completely different from conventional search techniques. However, the penalty coefficient increases the complexity of the search procedure. The choice of penalty coefficient in each iteration is the key issue, whether you use conventional methods or evolutionary algorithms, in penalty function based methods. There are three general categories in GAs to setting the penalty coefficient: the static one where the coefficient is a constant, dynamic where the coefficient is a predetermined monotonically nondecreasing sequence and adaptive which relies on population information to adjust the coefficient adaptively during the optimization process ${ }^{(21)(22)}$. The dynamic penalty assumed that $\mu_{k}=(C k)^{\alpha}$, where $C$ and $\alpha$ are constants. A reasonable choice for these parameters is $C=0.5$, and $\alpha=2$. This method requires a much smaller number (independent of the number of constraints) of parameters than the first method. Also, instead of defining several levels of violation, the pressure on infeasible solutions is increased due to the $(C k)^{\alpha}$ component of the penalty term: towards the end of the process (for high values of the generation number $k$ ) this component assumes large values.

The outputs of the algorithm were not satisfactory even for small problems with 3 sources, 2 transshipment points and 3 destinations. We run the algorithm 30 times, each time for 300 generations. Most of the time, the algorithm produces too many infeasible solutions (sometimes all) and ended up either with infeasible 
or suboptimal (very poor feasible) solutions. To overcome this problem, we modified the algorithm to force the feasibility of offspring in each and every generation. We introduce the modified algorithm as Modified GA (MGA).

\subsection{Algorithm 2: Modified GA}

- Population size 50

- Initial population was generated randomly ensuring their feasibility (discarding infeasible solutions).

- Heuristic crossover (as Algorithm 1).

- Probability of crossover $=1.0$.

- Constraints handling: ensure feasibility with repairing mechanism.

- Selection for crossover: First rank all the individuals based on the objective function values. Then select randomly one from the top 10 and two from the rest. Crossover was made between the first one and the better of the other two.

- Crossover: Once the parents are selected for crossover, choose a variable randomly (for example, a path or supply amount from a source to a transshipment point) and perform crossover. If the variable is within the current range (supply and capacity), we accept and reset the available supply and capacity for other variables. Otherwise, we set them at their extreme values. Once the supply is exhausted from a source, all unassigned paths from that source will be assigned to zero shipments without performing crossover operation. If any positive amount is left in any source after assigning all the paths (originating from that source) except one, then the entire amount will be allocated to that path without performing any crossover if it is less than the remaining transshipment point capacity. Otherwise the minimum of available supply and transshipment point capacity will be allocated.

- No mutation is used as the usual mutations would destroy the feasible structure of the current problem and we are not interested to design a specialized one at this stage.

- Ensuring Feasibility: After performing crossover following the above procedure, the problem could be infeasible in some cases because of the allocation without crossover as mentioned above and zero allocation after crossover (if the allocation is less than or equal to zero after crossover). These sorts of allocations may violate the supply constraints. Similar violation may occur in the destination side. In such a case, we apply a repair mechanism to ensure the feasibility in the final allocation.

- Repair Mechanism: After crossover, identify all the infeasible individuals. Identify the sources that have supplied less than their available amounts and determine the unassigned amounts that are available for supply. Generate a list of transshipment points which can receive more supplies. Randomly select a transshipment point from that list and select a source with unassigned goods, and then assign to the transshipment point as much as possible. Update the list of transshipment points and sources, and their unassigned supply/capacity. Continue this process until all supplies are exhausted. The same procedure will be applied to the second stage (transshipment point - destination) of the problem to remove infeasibility.

3.3 Algorithm 3: Modified $^{2}$ GA The Modified GA was further modified by changing the process of initial generation which would provide a better quality initial population. The process can be described as follows.

I. Set a list of sources with positive supply and destinations with unfulfilled demand.

II. Select a source (/destination) at random from the list, and find the cheapest route linked to transshipment points from that source (/destination). Any tie will be broken arbitrarily. Assign the maximum possible amount to that route. Update the involved supply (/demand) and available capacity of transshipment point. If the supply is exhausted (/demand is fulfilled), then the source (/destination) would be removed from the list.

II. Repeat II until all supplies are exhausted and requirements are fulfilled.

\section{Computational Experiences}

Three instances of a small problem (with 3 sources, 2 transshipment points and 3 destinations) were solved and the performances of the above two algorithms (SGA and MGA), in term of quality of solutions, were compared. The algorithms were run for 30 times and were allowed to simulate 300 generations in each run.

$>$ From the experimental results, it was found that MGA performs better for a transshipment problem. Most of the constraints in the test problems are tight constraints. As a result, it is very hard for SGA to find a reasonable number of feasible offspring in any generation which contributes to the generation of infeasible offspring in the subsequent generations. Although we allow infeasible offspring to be parents in many situations for better convergence, it does not seem to be effective when dealing with too many tight constraints ${ }^{(21)(22)}$. On the other hand, MGA ensures feasibility in every generation.

We then experimented with different sizes of test problems using the MGA algorithm. The test problems were generated by varying the number of sources, transshipment points and destinations as shown in Table 1, and the capacity and demand data were generated arbitrarily random fulfilling the assumptions (i) the total supply equals total demand (balanced problem) and (ii) the total capacity of the transshipment points is greater than the total supply. The unbalanced problems can also be solved using the same GA either (i) by making the problem balanced with a dummy source or destination as required or (ii) by allowing a minor change in the algorithm. The number of variables and the number of constraints involved in the mathematical models of the test problems are also shown in Table 1.

Although the quantity transported (decision variables of the model) requires integer values, the developed model can be treated as a linear program because of 
Table 1. The test problem details

\begin{tabular}{|c|c|c|c|c|c|}
\hline & \multicolumn{5}{|c|}{ Number of } \\
\hline $\begin{array}{c}\text { Problem } \\
\text { no. }\end{array}$ & Sources & $\begin{array}{c}\text { Trans- } \\
\text { shipments }\end{array}$ & $\begin{array}{c}\text { Destin- } \\
\text { ations }\end{array}$ & $\begin{array}{c}\text { Varia- } \\
\text { bles }\end{array}$ & $\begin{array}{c}\text { Const- } \\
\text { ranits }\end{array}$ \\
\hline \hline Prob-1 & 3 & 2 & 3 & 12 & 10 \\
Prob-2 & 4 & 2 & 4 & 16 & 12 \\
Prob-3 & 5 & 3 & 5 & 30 & 16 \\
Prob-4 & 8 & 4 & 8 & 64 & 24 \\
Prob-5 & 10 & 5 & 10 & 100 & 30 \\
Prob-6 & 12 & 7 & 12 & 168 & 38 \\
Prob-7 & 15 & 10 & 15 & 300 & 50 \\
Prob-8 & 20 & 15 & 20 & 600 & 70 \\
\hline
\end{tabular}

its special structure ${ }^{(16)}$. The model, when solving as linear program, would ensure integer values for the variables, provided supply, capacity and demand data are integer, without having any integrality condition in the model. We have solved the corresponding linear programming model using a commercial optimization package LINDO/LINGO. The linear program's objective function value and the outputs from GA are reported in Table 2. We have experimented on a Pentium4, $1.8 \mathrm{GHz}$, 256MB RAM, IBM PC. The GA Overallbest column indicates the best objective function value obtained out of 30 individual runs. The individual GA run was terminated when the best objective function value, obtained from that run, was repeated in five consecutive generations. We have not observed any improvement changing the stopping criteria from five to ten generations.

$>$ From columns 3 and 4 in Table 2, it is clear that MGA provides optimal solutions for smaller problems (problem number 1 and 2) but the quality of solutions deteriorates with the increase of search spaces (/larger problems). The solutions of the larger problems are not acceptable due to high percentage deviation (see column 5) from the optimal solution. It is more likely to have a better coverage for smaller search space (smaller problems) when we use a fixed population size for all problems.

It was observed that the quality of final solutions is dependent on the quality of the initial population. In order to improve the quality of solutions, we have conducted further experiments by changing the process of initial generation which is recognized as $M^{2} G A$ in the previous section. As shown in column 2 of Table 3, the results of $M^{2} G A$ are little improved (as compared to MGA, column 5 of Table 2) although the quality of solutions is still deteriorating with the increase of the search spaces.

4.1 Population Sizes We run $M^{2} G A$ by varying the population sizes as higher population size may be required for better coverage of the search space in case of larger problems. The percentage deviations of the objective function values obtained, from their respective optimal solutions, for population sizes 50, 500, 1000 and 2000 are presented in Table 3. The percent deviations of the average of best values obtained from 30 runs are also shown in Table 4 .

$>$ From Table 3, it is clear that the GA is able to provide optimal solutions for two smaller problems (Prob-1 and 2) with population size 50, and for Prob-3 and 4
Table 2. Comparing GA solutions with optimal solutions

\begin{tabular}{|c|c|c|c|c|}
\hline $\begin{array}{c}\text { Problem } \\
\text { no. }\end{array}$ & $\begin{array}{c}\text { Size } \\
(\mathrm{V} \times \mathrm{C})^{*}\end{array}$ & $\begin{array}{c}\text { Optimum } \\
(\text { LINDO }) \\
(\mathrm{a})\end{array}$ & $\begin{array}{c}\text { GA } \\
\text { overall } \\
\text { best (b) }\end{array}$ & $\begin{array}{c}\text { Percent } \\
\text { difference } \\
=100^{*}(\mathrm{~b}-\mathrm{a}) / \mathrm{a}\end{array}$ \\
\hline \hline Prob-1 & $12 \times 10$ & 32200 & 32200 & 0.00 \\
Prob-2 & $16 \times 12$ & 63500 & 63500 & 0.00 \\
Prob-3 & $30 \times 16$ & 62500 & 63000 & 0.80 \\
Prob-4 & $64 \times 24$ & 147200 & 148863 & 1.13 \\
Prob-5 & $100 \times 30$ & 141700 & 161800 & 14.18 \\
Prob-6 & $168 \times 38$ & 86750 & 112600 & 29.80 \\
Prob-7 & $300 \times 50$ & 91300 & 125700 & 37.68 \\
Prob-8 & $600 \times 70$ & 172500 & 244300 & 42.45 \\
\hline
\end{tabular}

Table 3. Percentage difference between best GA solution and optimal for different population sizes

\begin{tabular}{|c|c|c|c|c|}
\hline & \multicolumn{4}{|c|}{ Population Size } \\
\hline Problem & 50 & 500 & 1000 & 2000 \\
\hline \hline Prob-1 & 0.00 & 0.00 & 0.00 & 0.00 \\
Prob-2 & 0.00 & 0.00 & 0.00 & 0.00 \\
Prob-3 & 0.80 & 0.00 & 0.00 & 0.00 \\
Prob-4 & 1.13 & 0.00 & 0.00 & 0.00 \\
Prob-5 & 14.18 & 5.58 & 3.32 & 0.78 \\
Prob-6 & 29.80 & 7.44 & 5.76 & 1.10 \\
Prob-7 & 37.68 & 6.68 & 8.82 & 3.61 \\
Prob-8 & 42.45 & 12.94 & 10.35 & 4.52 \\
\hline
\end{tabular}

Table 4. Percentage difference between the average of best GA solutions and optimal for different population sizes

\begin{tabular}{|c|c|c|c|c|}
\hline & \multicolumn{4}{|c|}{ Population Size } \\
\hline Problem & 50 & 500 & 1000 & 2000 \\
\hline \hline Prob-1 & 0.00 & 0.00 & 0.00 & 0.00 \\
Prob-2 & 0.00 & 0.00 & 0.00 & 0.00 \\
Prob-3 & 1.90 & 0.45 & 0.08 & 0.00 \\
Prob-4 & 1.65 & 0.43 & 0.27 & 0.24 \\
Prob-5 & 20.56 & 8.39 & 7.42 & 5.04 \\
Prob-6 & 40.53 & 10.94 & 9.96 & 6.71 \\
Prob-7 & 55.47 & 16.21 & 14.62 & 10.69 \\
Prob-8 & 70.52 & 28.36 & 20.08 & 16.24 \\
\hline
\end{tabular}

with population size 500. As Table 3, for any given population size, it has shown that the quality of solutions deteriorates with the increase of problem sizes (Prob-3 to 8), and for any problem size, the quality of solutions improves with the increase of population sizes. When the population size is increased from 50 to 500, that means ten times higher, the quality of solutions improves a lot. The change of population sizes from 500 to 1000 or 1000 to 2000 (that is only two times) shows slow improvement. The improvements are more and more appreciable with the increase of problem sizes.

The percent improvements, based on the average of best values, are highly correlated with the population sizes. In Table 2 , the improvement for problem 7 is better with population size 500 than 1000 . However it is due to one run (out of 30 runs) only as we can see this not the case for average performances (see Table 3 and 4). From Tables 2, 3 and 4, one can easily conclude that the quality of solutions is highly dependent on the population size specifically when the search space is larger.

Although we assumed that the population size of 3000 would be excessive to the test problems under consider- 
ation, it helped to uncover very fascinating information. It improved the quality of solutions for test problems 6 and 8 significantly. That made us curious to examine the results with the population size of 4000 . This time, only problem 6 got better fitness values. That means it may not converge monotonically to the optimum fitness value just by simply increasing the population sizes. In other words, there exists an optimal population size for each instance of the problem when the search operators and selection pressure are fixed. Now the question is why GAs behave like this? Higher population sizes, by providing better diversity, help to reach better solutions. However, if the selection pressure and search operators do not appropriately match with the population size, the whole population prematurely converge to either a local optima or a sub-optimal region. That was the case in our experiments as our current experiments with changing selection pressure supports this fact. The solutions can be further improved by appropriately choosing the selection process.

4.2 Computational Time The average computational times per run, recorded in seconds, are shown in Table 5. The relationships seem to be polynomial.

Because of the special structure of the mathematical model, the model can be solved as a linear program. For this reason, we think, it is not fair to compare the computational times of GA with that of linear program. However for the readers, we like to mention here that the time taken by the optimization package is about one second for problems 6,7 and 8 .

As per our experimental results, the average number of generations required per run, for any test problem, decreases with the increase of population sizes. That supports the fact that the computational time may follow some sort of polynomial patterns. To give an idea about the fitness values in individual runs and the number of generations required by each individual run for solving the problem number 7 with four different population sizes are shown in Figures 1 and 2 respectively. From the figures, it is clear that both the fitness function and the number of generation required, with smaller population size, fluctuates a lot from run to run. However, there is no clear correlation between the quality of average fitness value and the number of generation required when compared run by run for each population size.

Table 5. Computational Time required (seconds) for different test problems with different population sizes

\begin{tabular}{|c|c|c|c|c|}
\hline & \multicolumn{4}{|c|}{ Population Size } \\
\hline Problem & 50 & 500 & 1000 & 2000 \\
\hline \hline Prob-1 & 0.019 & 0.470 & 1.560 & 4.620 \\
Prob-2 & 0.047 & 0.822 & 1.569 & 4.847 \\
Prob-3 & 0.088 & 0.926 & 2.246 & 5.736 \\
Prob-4 & 0.234 & 1.679 & 3.636 & 8.247 \\
Prob-5 & 0.493 & 3.988 & 7.376 & 17.348 \\
Prob-6 & 10.489 & 22.914 & 39.015 & 70.971 \\
Prob-7 & 18.080 & 93.108 & 178.886 & 320.392 \\
Prob-8 & 54.531 & 489.511 & 691.878 & 993.056 \\
\hline
\end{tabular}

4.3 Number of Fitness Evaluations The average number of fitness evaluated can be calculated as the population size times the average number of generations required for that population size. The ratios of the number of fitness evaluations (taking the population size 50 as the datum) for different population sizes are given in Table 6 .

Consider the Prob- 8 with population size 2000. Although the increase of population size from 50 to 2000 is 40 times higher, the average number of fitness evaluations is only 12.36 times higher which is a favorable point for using higher population size when necessary. We must mention here that the relaxation of stopping criteria (for example allowing more generations) with smaller population sizes does not improve the fitness value.

To ensure the quality within a certain level, the use of population size of 2000 or more for a problem of few hundreds variables and less than 50 constraints would not be a convincing approach to many practitioners. However one should not forget that we have not ensured the best crossover and selection process for solving this problem.

4.4 Overall GA Solutions The overall GA solutions when run up to population size of 4000 are shown in Table 7.

We believe by changing the selection pressure and other parameters used in running the GA, we would able to improve the performance of the designed algorithm.

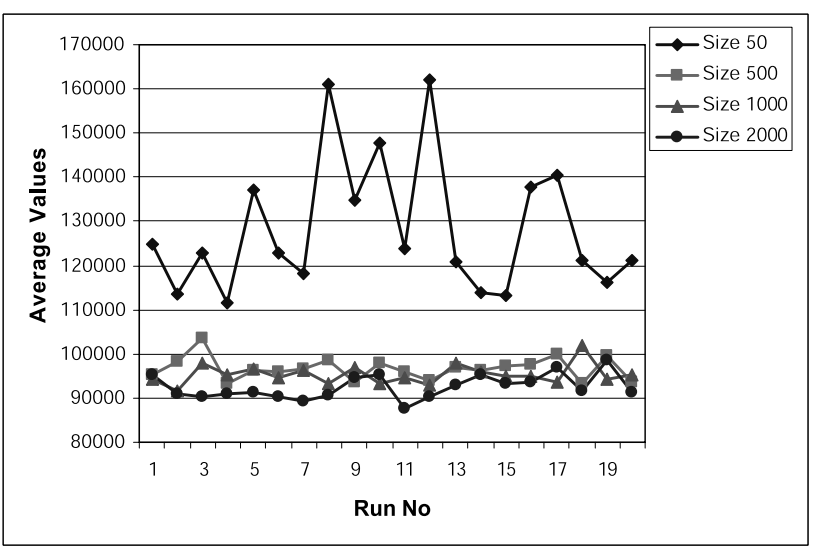

Fig. 1. Average fitness in individual run

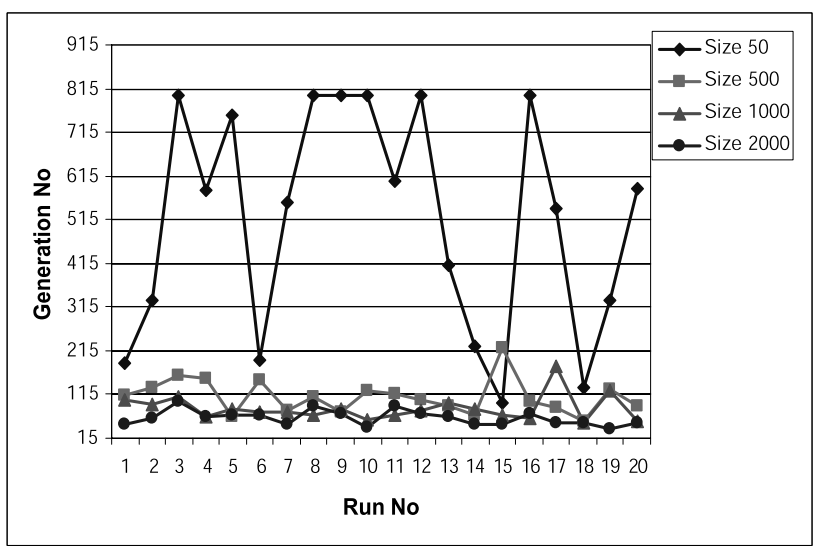

Fig. 2. The number of generation required per run 
Table 6. Comparison of the number of fitness evaluations as ratio of different population sizes

\begin{tabular}{|c|c|c|c|c|}
\hline & \multicolumn{4}{|c|}{ Ratio of population Sizes } \\
\hline Problem & $50: 50$ & $500: 50$ & $1000: 50$ & $2000: 50$ \\
\hline \hline Prob-3 & 1.00 & 9.33 & - & - \\
Prob-4 & 1.00 & 6.29 & - & - \\
Prob-5 & 1.00 & 7.60 & 12.68 & 24.03 \\
Prob-6 & 1.00 & 3.96 & 5.93 & 9.11 \\
Prob-7 & 1.00 & 4.85 & 7.66 & 11.88 \\
Prob-8 & 1.00 & 6.35 & 8.31 & 12.36 \\
\hline
\end{tabular}

Table 7. Comparing GA with optimal solutions

\begin{tabular}{|c|c|c|c|c|}
\hline $\begin{array}{c}\text { Problem } \\
\text { no. }\end{array}$ & $\begin{array}{c}\text { Optimum } \\
\text { (LINDO) } \\
\text { (a) }\end{array}$ & $\begin{array}{c}\text { GA } \\
\text { overall } \\
\text { best (b) }\end{array}$ & $\begin{array}{c}\text { Percent } \\
\text { difference } \\
100 *(b-a) / a\end{array}$ & $\begin{array}{c}\text { Corres- } \\
\text { ponding } \\
\text { Pop-size }\end{array}$ \\
\hline \hline Prob-1 & 32200 & 32200 & 0 & 50 \\
Prob-2 & 63500 & 63500 & 0 & 50 \\
Prob-3 & 62500 & 62500 & 0 & 500 \\
Prob-4 & 147200 & 147200 & 0 & 500 \\
Prob-5 & 141700 & 142800 & 0.7763 & 2000 \\
Prob-6 & 86750 & 87700 & 0.3500 & 4000 \\
Prob-7 & 91300 & 94600 & 3.6145 & 2000 \\
Prob-8 & 172500 & 180297 & 0.6100 & 3000 \\
\hline
\end{tabular}

\section{Conclusions}

In this paper, we introduced a transshipment problem and formulated its mathematical model. We developed three versions of GA for solving the developed mathematical model and compared their solutions.

We did experiments by changing the problem size and the population size. It is generally known that the quality of solutions improves with the increase of population sizes. This research confirms this general finding once again but it is restricted up to a certain level of population size when the search operators and selection pressure are fixed. As experimented in this research, this is true for a case of two-stage transportation problem. In addition it shows that it is necessary to increase the population size when the search space is larger. Although it generally dictates that the higher population results in very high number of fitness evaluations (and high computational time), the real number of fitness evaluated (and the computational time required), when increasing the population sizes, is much lower than expected. However, it is an open question how to choose a population, which would ensure a certain quality level, for a given instance of a problem. To answer this question one needs to involve the problem size and complexity, population size and all other methods and parameters involved with the GA to be applied. For unsolved largescale optimization problems, it is hard to sense whether the algorithm has reached to its optimal solution or not, even though the algorithm converges nicely to a solution (either local or global). As a result, it is a very difficult task to find a general equation for population sizes to be used.

(Manuscript received Oct. 1, 2003,

revised June 15, 2004)

\section{References}

(1) C.W. Ahn and R.S. Ramakrishna: "A Genetic Algorithm for Shortest Path Routing Problem and the Sizing of Populations", IEEE Trans on Evolutionary Computation, Vol.6, pp.566-579 (2202)

( 2 ) T. Back and H-P. Schwefel: "An Overview of Evolutionary Algorithms for Parameter Optimization", Evolutionary Computation, Vol.1, pp.1-24 (1993)

(3) W. Barnett, C. Chiarella, S. Keen, R. Marks, and H. Schnabl: Complexity and Evolution, Cambridge University Press (2000)

(4) L. Davis: Handbook of Genetic Algorithms, Van Nostrand Reinhold, New York, (1991)

(5) A. Eiben, R. Hinterding, and Z. Michalewicz: "Parameter Control in Evolutionary Algorithms", IEEE Transactions on Evolutionary Computation, Vol.3, pp.124-141 (1999)

(6) M. Fischer and Y. Leung: GeoComputational Modelling Techniques and Applications, Springer-Verlag, Berlin, (2001)

( 7 ) D. Fogel and L. C. Stayton: "On the Effectiveness of Crossover in Simulated Evolutionary Optimization", ByoSystems, Vol.32, pp.171-82 (1994)

( 8 ) M. Gen, K. Ida, and Y. Li: "Bicriteria transportation problem by hybrid genetic algorithm", Computers and Industrial Engineering, Vol.35, pp.363-366 (1998)

(9) M. Gen, K. Ida, and Y. Li: "Solving bicriteria solid transportation problem by genetic algorithm", IEEE International Conference on Systems, Man, and Cybernetics, pp.12001207 (1994)

(10) D. Goldberg: Genetic Algorithms in Search, Optimization, and Machine Learning, Addison-Wesley, Reading, MA, (1989)

(11) D. Goldberg, K. Deb, and J.H. Clark: "Genetic Algorithms, Noise, and the Sizing of Populations", Complex Systems, Vol.6, pp.333-362 (1992)

(12) D. Goldberg and M. Rudnick: "Genetic Algorithms and the Variance of Fitness", Complex Systems, Vol.2, pp.265-278 (1988)

(13) J. Gottlieb and L. Paulmann: "Genetic algorithms for the fixed charge transportation problem", IEEE World Congress on Computational Intelligence, pp.330-335 (1998)

(14) G. Harik, E. Canti-Paz, D. Goldberg, and B.L. Miller: "The Gambler's Ruin Problem, Genetic Algoriths, and the Sizing of Populations", Evolutionary Computation, Vol.7, pp.231-253 (1999)

(15) J. Holland: Adaptation in Natural and Artificial Systems, University of Michigan Press, Ann Arbor, MI, USA, (1975)

(16) F. Hillier and G. Lieberman: Introduction to Operations Research, McGraw-Hill, Boston, USA, (2001)

(17) D. Jiang, W. Du; X. Chen: "GA based location models for physical distribution centers", IEEE International Conference on Intelligent Processing Systems, pp.553-557 (1997)

(18) I. Karanta, T. Mikkota, C. Bounsaythip, O. Jokinen, and J. Savola: "Genetic algorithms applied to a wood collection problem", IEEE International Conference on Systems, Man, and Cybernetics, pp.635-639 (1999)

(19) Z. Michalewicz: Genetic Algorithms + Data Structures = Evolution Programs, 3rd ed., New York, Springer-Verlag, (1996)

(20) M. Sakawa and K. Kato: "Genetic Algorithms with Double Strings for 0-1 Programming Problems", European Journal of Operational Research, Vol.144, pp.581-597 (2003)

(21) R. Sarker, T. Runarsson, and C. Newton: "Genetic Algorithms for Solving A Class of Constrained Nonlinear Integer Programs", International Transaction in Operational Research, Vol.8, pp.61-74 (2001)

(22) R. Sarker, T. Runarsson, and C. Newton: "A Constrained Multiple Raw Materials Manufacturing Batch Sizing Problem", International Transaction in Operational Research, Vol.8, pp.121-138 (2001)

(23) A. Syarif and M. Gen: "Double Spanning Tree-Based Genetic Algorithm for Two Stage Transportation Problem", Int. J. of Knowledge-Based Engineering Systems, Vol.7, pp.157-163 (2003)

(24) A. Syarif, Y. Yun, and M. Gen: "Study on multi-stage lo- 
gistic chain network: a spanning tree-based genetic algorithm approach", Computers and Industrial Engineering, Vol.43, pp.299-314 (2002)

(25) A. Quintero and S. Pierre: "Evolutionary Approach to Optimize the Assignment of Cells to Switches in Personal Communication Networks", Computer Communications, Vol.26, pp.927-938 (2003)

(26) S.R. Thangiah and K.E. Nygard: "MICAH: a genetic algorithm system for multi-commodity transhipment problems", 1992 Proceedings of the Eighth Conference on Artificial Intelligence for Applications, pp.240-246 (1992)

(27) G.A. Vignaux and Z. Michalewicz: "A genetic algorithm for the linear transportation problem", IEEE Transactions on Systems, Man and Cybernetics, vol.21, pp.445-452 (1991)

(28) X. Yao (edited): Evolutionary Computation: Theory and Application, World Scientific Publ. Co., Singapore (1999)

(29) X. Yao: "Evolutionary Computation: A Gentle Introduction", In Evolutionary Optimization, R. Sarker, M. Mohammadian and X. Yao (edited), Kluwer, USA, pp.27-56 (2002)

Ruhul Sarker (Non-member) Ruhul Sarker received his

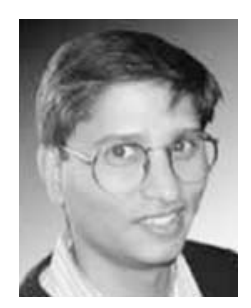
Ph.D. in 1991 from DalTech, Dalhousie University, Halifax, Canada, and is currently a senior lecturer in the School of Information Technology and Electrical Engineering, University of New South Wales, ADFA Campus, Canberra, Australia. His main research interests are Evolutionary Optimization, Neural Networks and Applied Operations Research. He has recently edited four books and has published more than 90 refereed papers in international journals and conference proceedings. Dr Sarker is actively involved with a number of national and international conferences \& workshop organizations in the capacity of chair, co-chair or program committee member. He has recently served as a technical co-chair for IEEECEC2003.
Akira Namatame (Member) Akira Namatame received his

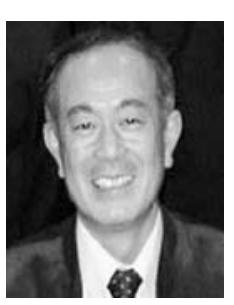
Ph.D. from Stanford University in 1979. He joined the Department of Defense of Japan, where he was the $\mathrm{R} \& \mathrm{D}$ program manager of several electronic defense systems. He joined the Department of Computer Science at Japan Defense Academy in 1988. He was a visiting Professor at George Mason University from 1989 to 1990 . He is now professor and chair of the Department of Computer Science. His research interests include multi-agents, evolutionary computation, game theory, and computational economics. He has published more than 160 refereed journal and conference papers. He also published three books. 